\title{
MULTI-REGIME INTEGRATED TRANSDUCER NETWORKS
}

\author{
S.C. Jacobsen, M. Olivier, B.J. Maclean, M.G. Mladejovsky and M.R. Whitaker \\ Sarcos Research Corporation, 360 Wakara Way, Salt Lake, UT 84108 \\ Center for Engineering Design, University of Utah, Salt Lake UT 84112.
}

\begin{abstract}
The approach described in this paper has been used to design and manufacture a variety of sensors and networks for the measurement of: strain, rotation, displacement, pressure, vibration, flow, multi-axis fluid shear, multi-axis strain, touch, multi-axis acceleration, and sound. This paper discusses four example systems.

System State Sensors

- UniAxial Strain Transducer

Rotational Displacement Transducer

(UAST)

Fluid State Sensors

- Fluid Shear Transducer

Pressure Transducer Network

\section{INTRODUCTION}

Beginning in 1982, Sarcos Incorporated and the Center for Engineering Design at the University of Utah became involved in an increasing number of projects focused on the development of robots and other sensor-intensive machines. For example, one robotics project for Disney used thousands of high resolution rotation and strain sensors. A typical robot included 50 Degrees-of-Freedom (DOF) and required approximately 90 sensors and 50 actuators. Entertainment robotics projects alone have required over 4000 sensors and 2000 actuators. In these applications, sensors have accounted for up to 30 percent of system cost, and up to half of the system failures have occurred in the supporting wiring harnesses and connectors. Sensors and wiring assemblies have been primary drivers of cost and reliability in other similar applications such as undersea tele-robots, prosthetic limbs, and body motion capture systems (where wiring is routed across moving structures and sensors reside in harsh environments).

In the early 80's, Sarcos began investigating new approaches to sensor and network design [1-5]. Specifically required were robust systems with: high resolution, absolute digital multiplexed output, small size, and low cost. Of main interest were the multiplexed systems which could reduce the wire counts necessary to support interconnected groups of multi-regime sensors

In 1986 a first proposal was submitted to DARPA entitled "Micro Electro Mechanical Systems" (MEMS). In that project, and others later funded by DARPA, NASA, NAVSEA, and commercial sponsors, a number of approaches were investigated. Sensors are emerging from those development efforts and are now a part of preliminary application efforts in aircraft (F18, F15, C141, C130), helicopters (AH-64 and UH60 ), submarines (LSV, RCM), railroad operation monitoring systems, robots, automotive, and structural systems.

\section{APPROACH}

The sensors defined herein are composed of two basic subsystems - (1) a planar silicon base which contains an array of many field detectors along with supporting electronics, and (2) a companion array of electrostatic field emitters residing on the surface of an adjacent planar armature made of quartz, sapphire, or glass. Base and armature surfaces interact in close proximity and can, depending on the configuration of emitter and detector arrays, measure specified relative movements between the armature and base with high resolution. Figure 1 shows base and armature structures used in the RDT

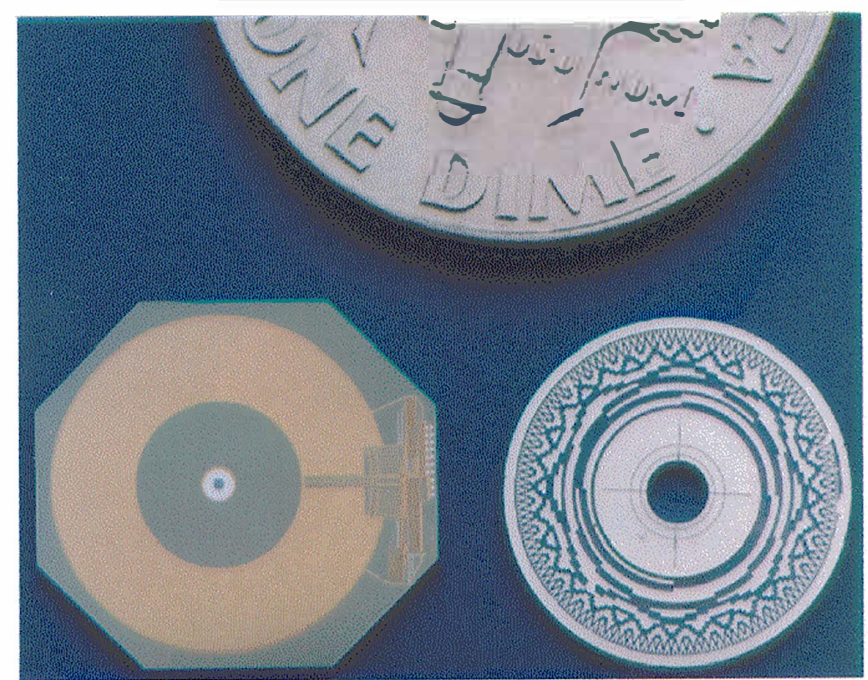

Figure 1. Base and armature structures used in the Rotary Displacement Transducer (RDT).

In operation, the gap between armature and base is small and is tightly maintained either by direct contact, between bearing surfaces created on the structures, or by a suspension system composed of adjacent bearings or flexures. In the four examples of this paper, relative movements are restricted to one or two DOF. In other cases relative movements can be up to six DOF.

Emitters and detectors which reside on armatures and bases are the fundamental measuring elements in each sensor. A detector (typically 20 to 150 by 50 to $150 \mu \mathrm{m}$ ) is a conductive region on the base which is connected to a local circuit that performs amplification and conversion functions. An emitter (typically 30 to 200 by 100 to $300 \mu \mathrm{m}$ ) is a conductive region on the armature which is driven either capacitively from the base, or by a connected source. The shape details of emitters and detectors are very different for various sensors.

Emitters and detectors are configured into arrays designed to be maximally sensitive to certain relative motions between base and armature, and minimally sensitive to other movements. On to the emitter array is impressed one or a group of signals which are then sensed by the detector array. Figure 2 schematically illustrates one simple arrangement of emitters, detectors, and circuits configured linearly with slightly different spacing to form a vernier arrangement. In the following section, a vernier array is discussed in more detail as the basis for the UniAxial Strain Transducer (UAST). In Figure 2, the top 
line of cross-hatched rectangles represents emitters in the moving armature and the bottom rectangle represents a detector, connected to circuits in the base. Figure 3 illustrates the signals created by an array of detectors driven by two sets of interleaved emitters and identical inverted waveforms (180 degrees out of phase). Note the beats in the interference pattern created by the overlay of the emitter array on the detector array.

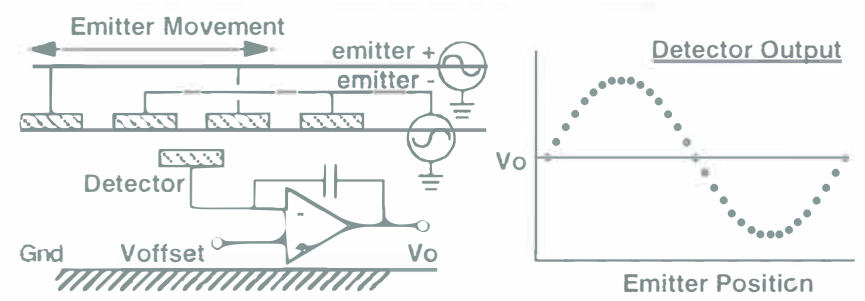

Figure 2. Schematic diagram showing emitters, single detector, and associated circuitry.
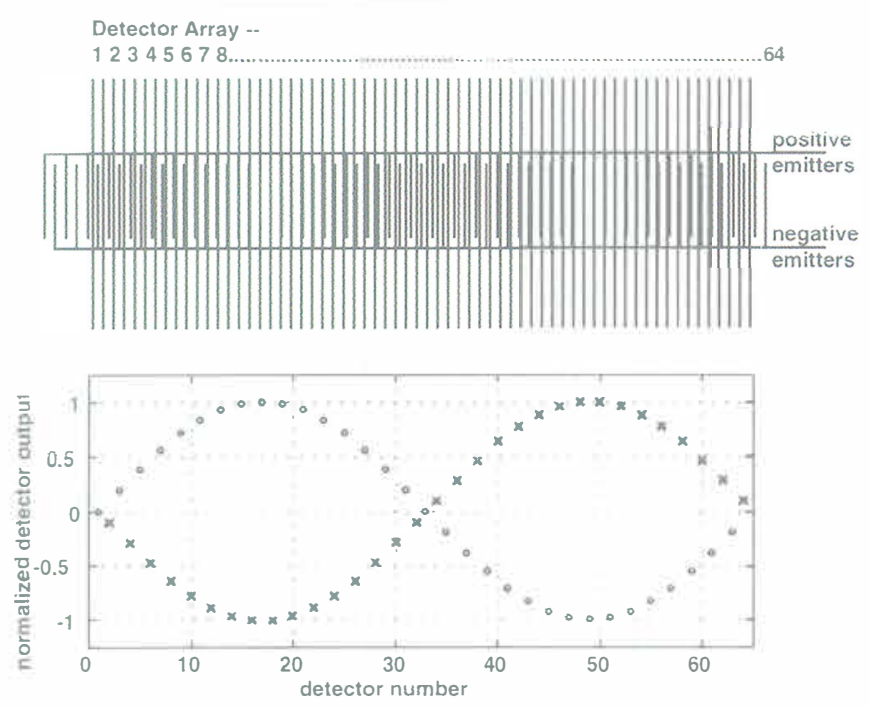

Figure 3. Schematic representation of signals from mulitple detectors in a vernier array. The vernier arrangement of field emitter and field effect detectors produces a periodic waveform which phase depends on the relative position between the armature and the base.

In the case of Fig. 3, the waveform, defined by plotting detector output against its spatial position, appears similar to a sinusoid with its phase shifting when the emitter array is translated past the detector array. In general, signals from the detector array are combined by local circuitry and processed to produce a digital signal which represents the relative position of base and armature. Digital signals are created by circuits on the base which operate very uniformly from detector to detector, are drift-free, and can be multiplexed onto a minimal-wire bus using various architectures to be discussed later.

Each sensor uses a "front end" which interacts with the physical environment to transform a measured parameter, such as rotation, strain, shear, or pressure, into a micro-movement of the armature which is converted to a signal by the base chip. Sensors include packages with pass-through mechanisms, or ports, which allow sealed interaction with the environment through the front-end and into the armature. Packages also include sealed conduits to pass wiring between individual sensor nodes and central processing modules.
Challenges of this approach include: defining array archirectures and signal processing schemes for each application, designing base chips with non-standard configurations of circuitry (non-orthogonal), creating suspension and/or on-chip bearing systems to maintain precise relationships between armatures and bases, defining multiplexing schemes which can be used successfully with existing systems, and designing packages with non-restrictive pass-throughs which are resistant to challenging environments.

\section{UNI-AXIAL STRAIN TRANSDUCER (UAST)}

\section{UAST - Objectives}

The UAST is part of a strain sensing network for applications such as: condition-based operation and maintenance, integrated load cells, large dynamic range scales, and others. Targeted characteristics include: (1) high resolution, (2) high strain operating range with high allowable overextension without damage (bangs), (3) refresh rates necessary for structural monitoring at lower frequency ranges (up to $1 \mathrm{kHz}$ ) and higher ranges for vibration monitoring (up to $20 \mathrm{kHz}$ ), (4) system architecture designed for use of up to 128 sensors on a digital network, (5) high vibration and impact tolerance for rugged applications, (6) shielding against electromagnetic fields, and (7) a package size and shape suitable for a broad range of uses.

UAST version 3 (UAST 3), shown in Fig. 4, was completed in 1997. Figures 5 and 6 show applications of UAST 3 on a half-scale F-18 scale model test apparatus, and on an AH-64 Apache Longbow helicopter rotor blade.

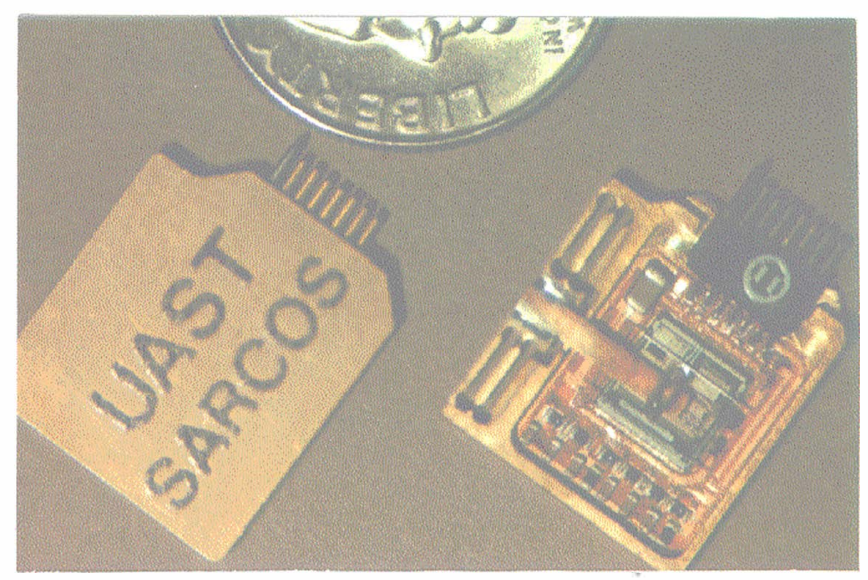

Figure 4. Photograph showing the UAST 3 package, base chip, quartz emitter, internal circuitry, and emitter drive flexure.

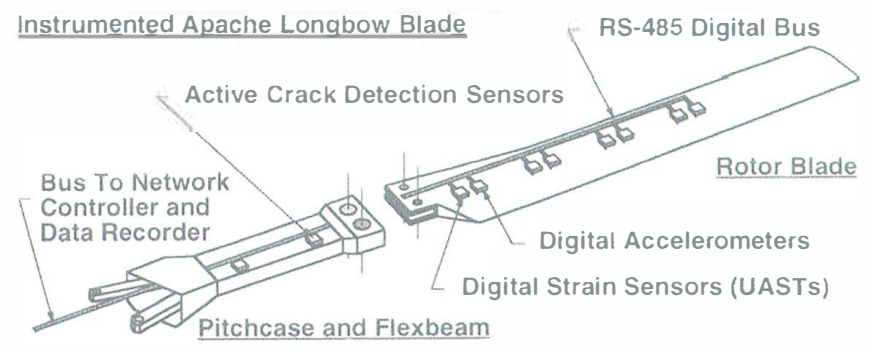

Figure 5. AH-64 Apache rotor blade equipped with UAST sensors used to monitor dynamic load cycles as well as crack growth. 


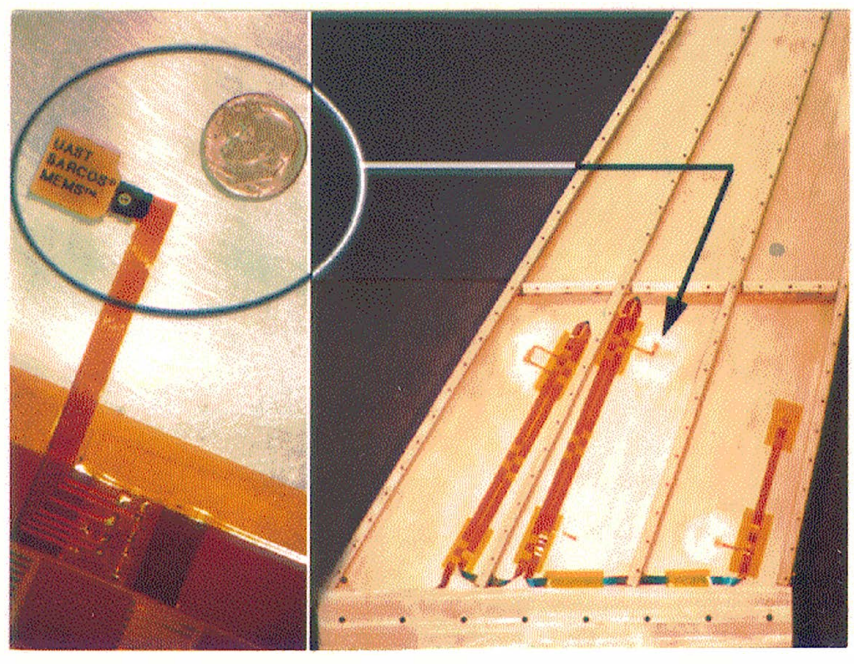

Figure 6. Half-scale F-18 vertical tail torque box showing a network of UAST 3 sensors used for prognostics and health monitoring.

\section{UAST - Configuration}

Figure 4 shows the UAST 3 package with dimensions of $12 \times 13.5 \times 2.5 \mathrm{~mm}$. The package material is beryllium-copper and the emitter support arm is assembled by a soldering process. The package includes three mounting pads on the housing bottom side. Two pads are static and one is movable via a double flexure suspension which is connected to a link which moves the armature over the base. The primary function of the package is to attach to the structure to be monitored, and pass strain displacements from the third attachment pad (the front end) to the armature which position is computed by the base. The package also contains four capacitors which provide the filtering required for the base chip operation.

The armature operates in direct contact with the base chip and is supported by a bearing surface which provides a clearance gap of approximately 2 to $5 \mu \mathrm{m}$ between the emitter and detector arrays. Although contact friction causes some hysteresis, resolution is sufficient to monitor strains down to 0.35 microstrain $(3.5 \mathrm{~nm}$ at $10 \mathrm{~mm}$ gage length) with high bandwidth and dynamic range. Note here that the UAST is really a micro linear extensometer and not a strain gage since relative displacement, not material distortion, is used to generate an output.

UAST 3 as applied in the configuration shown in Fig. 4, is not hermetically. An upcoming system, UAST 4, will include a flexion-based, sealed package, similar to that illustrated in Fig. 7. UAST 4 is also mounted via two base pads and a moving pad. Observe that one of the base pads is suspended by a transverse flexure which isolates the measurement from Poisson-induced deflections. The moving pad is supported by a beam and flexure assembly which passes through a membrane flexure into the inside of the package. The internal link segment, together with certain flexures, supports the quartz armature which contains the emitter array. In this design the emitter element moves over the base chip without contact.

An emerging development, the IC chip for UAST 5, will be designed in 0.6 micron CMOS and will include both intrinsic and computation-based temperature compensation, load cycle counting, new strain computation algorithm, power saving auto-sleep modes, high sample rates, smaller foot print, and multiple communication protocols.

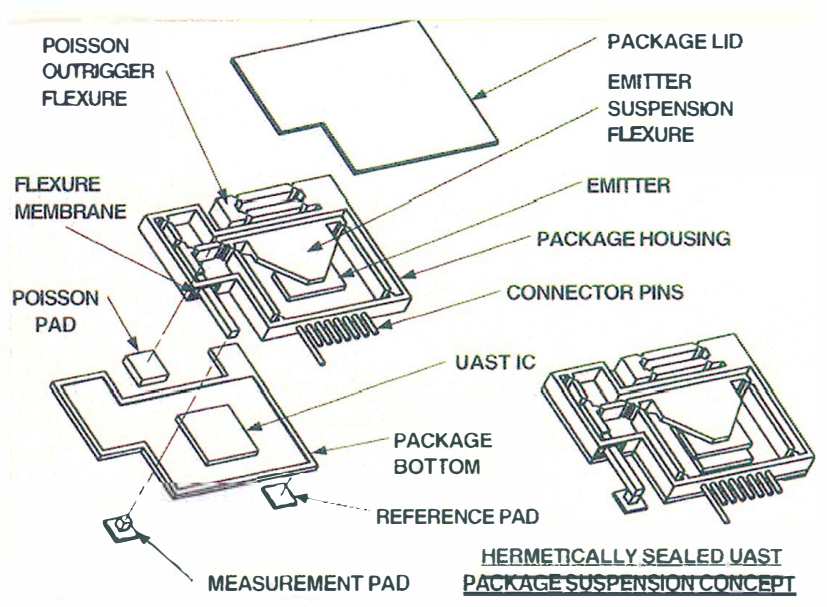

Figure 7. UAST version 4 package - hermetically sealed.

\section{UAST - Armature \& Base}

Figure 8 shows the major functional blocks of the UAST 3 base chip. As indicated earlier, the strain information is obtained by measuring the relative displacement between the base and the armature. The vernier arrangement between the emitter fingers and the detector array results in a quasisinusoidal waveform of detector output (see Fig. 3) in which the magnitude of the strain is encoded in the phase of the waveform.

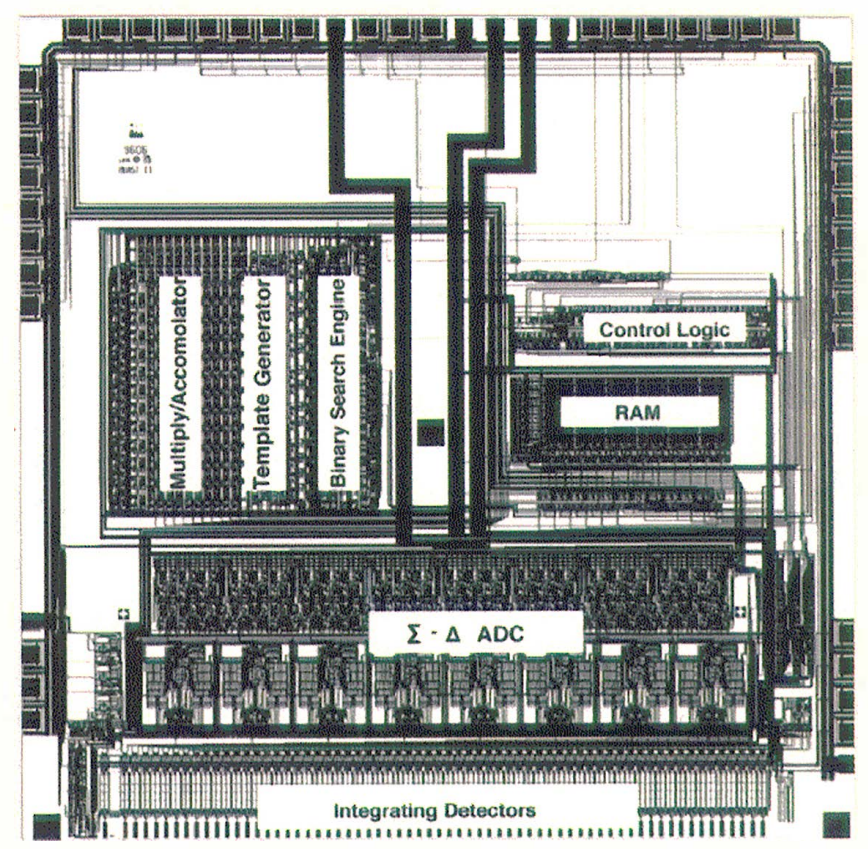

Figure 8. Layout of the UAST 3 base chip showing the major functional blocks.

The emitter array consists of two interleaved sets of conductive elements (lithographically patterned chromium film) driven with identical, reverse polarity, waveforms. The detector array elements, along with associated circuits, are fabricated in silicon using 1.2 micron CMOS design rules.

UAST 3's detector pads are 24 by $89 \mu \mathrm{m}$ with a centerline spacing of $55.8 \mu \mathrm{m}$. Emitter pads are 24 by $200 \mu \mathrm{m}$ with a centerline spacing of $57.6 \mu \mathrm{m}$ (which corresponds to an interference spatial wavelength of $115.2 \mu \mathrm{m}$ ). Note that the 
difference in spacing between emitter and detector array elements determines the interference-pattern wavelength as well as the sensitivity to phase translation of the waveform shown in Fig. 3.

To convert the output of 64 detectors into a single digital multiplexed output the following procedure is accomplished on the single chip shown in Fig. 8. First, a series of up to 16 strobe pulses are applied to the emitter fingers in order to record the waveform on the array of 64 charge-integrating amplifiers connected to the detectors. Next, the analog outputs of the detector array are fed to 8 parallel sigma-delta analog-to-digital converters (ADC) which share the task of digitizing the 64 detector values and placing the results in RAM. Each conversion requires $2^{(n+1)}$ clock pulses to create n-bits of detector resolution (supporting from 7 to 12 bits of conversion, depending on the UAST resolution mode selected). To start the strain computation algorithm, shown in Fig. 9, a 14-bit triangular waveform of 64 template values is generated with the same period as the detector data but with one of $2^{15}(32,768)$ phase positions. A convolution can then be performed by successively summing the products between the respective detector and template values at a given template position. When this is done for all 32,768 possible template locations, a periodic function is created whose phase corresponds to the relative position of the armature and base. For efficiency, the convolution is calculated only 15 times by executing a binary search to find the zero-axis crossing of the convolution waveform (this approach yields 15-bits of resolution between the armature and base when operating the ADCs with 12-bit conversions, i.e., 0.35 micro-strain for a $10 \mathrm{~mm}$ gage length). Note that all 64 detector values contribute to the final calculated strain (displacement) value, providing a robust form of spatial filtering which, incidentally, has no sensitivity to DC offsets in the detector waveform. This important attribute allows the UAST to calculate the same answer regardless of variations in the gap between the armature and base. Finally, the resulting digital strain number is multiplexed onto a common 5-wire bus (including power, ground, distributed $10 \mathrm{MHz}$ clock, token, and data) using the token passing method diagrammed in Fig. 10 (other methods using random addressing of UASTs on bidirectional RS-485 busses have also been developed for healthmonitoring applications on AH-64 and UH-60 helicopters).

\section{UAST - Performance}

Intrinsic to the operation of a UAST sensor network is the flexibility to trade sensor sample rate against UAST strain resolution, i.e., using fewer clock pulses to convert the detector values, speeds up the calculation of the strain value but with a corresponding decrease in strain resolution. The number of sensors on the network also impacts the sample rate since more sensors require more time to transmit their data which occurs at $2.5 \mathrm{Mbits} / \mathrm{sec}$. For example, 128 sensors operated in 15-bit mode (resolution of 0.35 micro-strain, i.e., $3.5 \mathrm{~nm}$ ) can be sampled at $131 \mathrm{~Hz}$ each, while 10-bit mode (11.25 micro-strain resolution) allows sampling at $786 \mathrm{~Hz}$ each. Likewise, a smaller number of 8 sensors can be operated in 15-bit and 10-bit modes while sampling at $148 \mathrm{~Hz}$ and $2452 \mathrm{~Hz}$, respectively [1]. Note that in the UAST, sampling across the detector array is accomplished simultaneously, in a snap-shot fashion. Likewise, via use of the token line, data is acquired synchronously across the entire network.
Along with the many attributes of the UAST detection scheme come design issues associated with integrating the base and armature into a viable transducer package which provides ease of installation and robust operation in harsh environments (vibration, temperature, moisture, electrical interference, shock, etc.). The simple UAST packaging schemes used thus far on proof-of-concept hardware platforms are currently being redesigned to provide hermeticity, low profile for embedability in composites, lower fabrication costs, and other performance aspects associated with near-term real world applications.

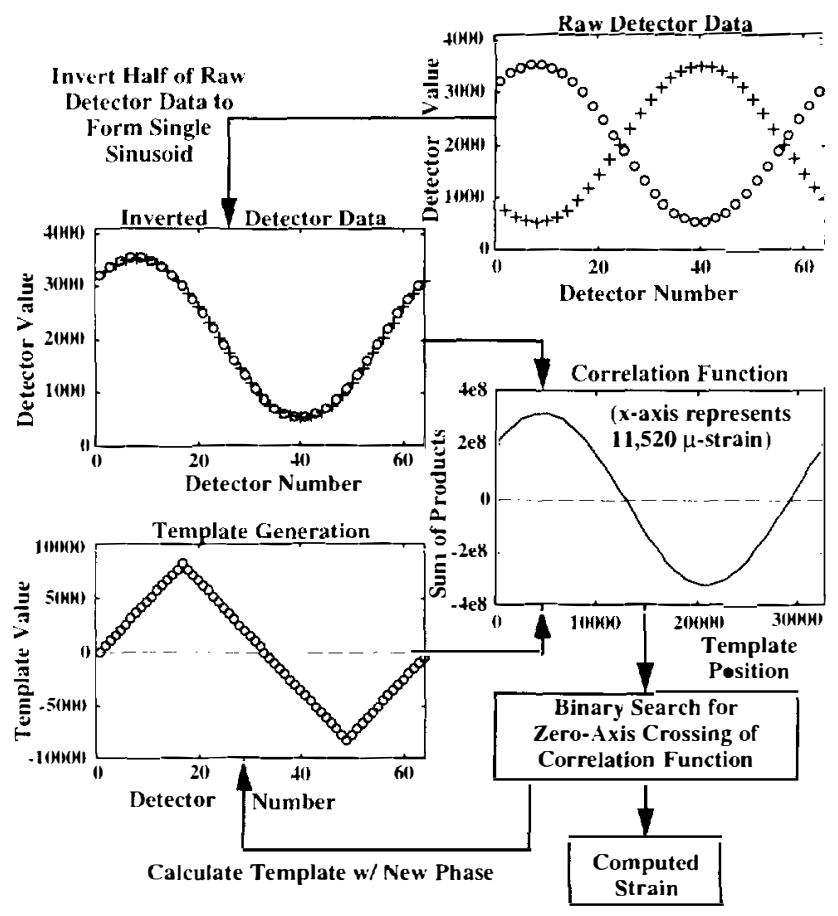

Figure 9. A binary search for the zero-axis crossing of a correlation function is formulated using the sum of products between the measured detector data and digitally synthesized template values.

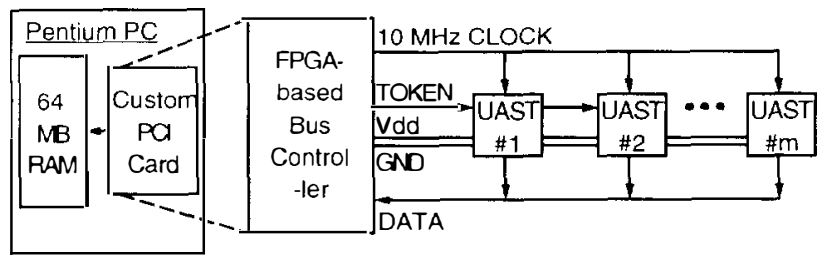

Figure 10. Multiplexing and token passing network scheme.

\section{ROTARY DISPLACEMENT TRANSDUCER}

\section{RDT - Objectives}

The RDT is a networked encoding systems for use in robotics and other applications where many DOF are monitored. Target characteristics include: (1) absolute encoding of angular position - to insure stability at startup; (2) resolution of 15 to 16 bits, upgradable to even higher resolutions - the present systems give 13 bits of resolution with prototypes already designed to achieve 15 bits resolution; (3) small size: 3 stacked dimes, (4) unshielded three-wire bus architecture capable of 
supporting up to 128 sensors at a rate of 400 samples/sec per sensor (fast enough to do real time dynamic compensation in small, fast actuators and to allow accurate computation of rotational velocity from dynamic position measurements).

\section{RDT - Configuration}

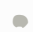

RDT version 2 (RDT 2) was designed to achieve 13 bits of resolution and was completed in 1995, with only a few minor problems. The armature and base chip for RDT 2 were shown earlier in Fig. 1. The basic elements of the RDT 2 package are shown below in Fig. 11. The components include: (1) shaft, spring-disk, and armature - which are connected in the assembled device; (2) housing with bearing tube and electrical connectors; (3) base chip which gets bonded to the housing and contains a $1 \mathrm{~mm}$ diameter hole for shaft pass-through. It is important to note that all electronic elements required for the operation of the sensor are fabricated as part of the base chip.

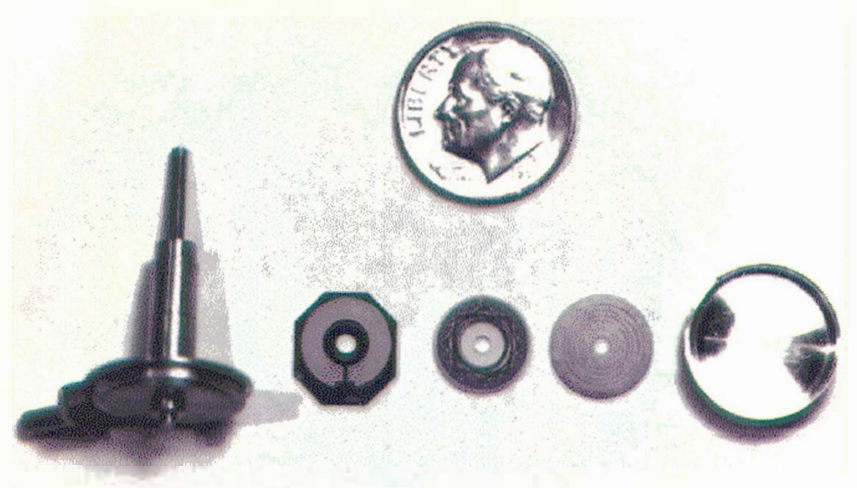

Figure 11. RDT 2 package components, showing, from left to right, the rotor shaft and housing, the base IC chip, the armature (rotor disk), the disk spring, and the housing cover.

The armature (rotor disk) and base IC chip are in direct contact at the center region of the base chip, where a throughhole has been drilled. A flat bearing surface at the center of the base is achieved by standard IC processing, and a hard passivation layer is used as the bearing surface. A small mesa, approximately $10 \mu \mathrm{m}$ high, is micromachined on the sapphire armature and provides a well-controlled gap between the emitter and the detector planes. At assembly, the gap between the base and the armature is filled with silicone oil to provide lubrication and to increase electrostatic coupling. The spring-disk (Fig. 11) is designed to be soft in the axial and tilt directions and stiff in rotation. The axial stiffness and preload of the spring are sufficient to maintain contact between the rotor disk and the base IC for acceleration at high g-levels while running. Figure 12 shows an industrial ruggedized package which is sealed, and features a 4-wire pass-through.

The RDT is designed to allow multiplexing of up to 128 sensors on a three-wire bus. The first wire provides power and data, the second wire provides reset, address increment and dataclock, and the third wire is the ground return. Each RDT contains an address register determined by wirebonds on seven pads of the base chip shown in Fig. 1. A pulse train from the bus first resets the entire network and then counts the register until a comparison is achieve at each RDT. When the comparison register agrees with the sequential address, a 16-bit serial data stream is impressed on the data line. The network thus produces time-staggered data which may impact or limit the type of control approach used, e.g., matrix-based or serial control.

Reset and address-increment are encoded on the three state clock line as a full $5 \mathrm{~V}$ amplitude pair of pulses or as a single pulse, respectively. Data bits are serially clocked out by an interleaved half amplitude clock pulse. The data bits are encoded on to the power line by modulating the current drawn by the sensor. An RS-232 interface has been implemented but various other interfaces to a digital controller can be easily developed.

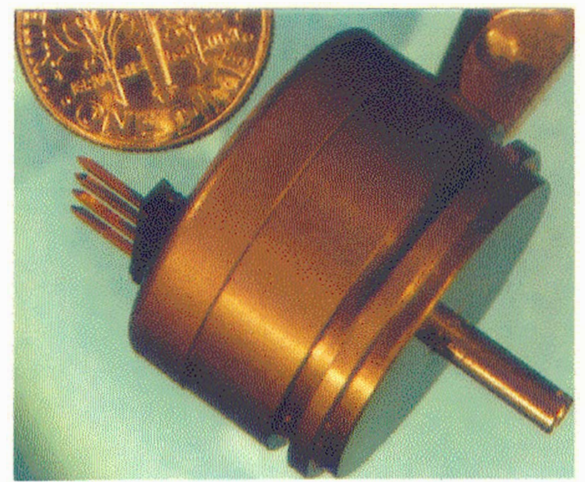

Figure 12. Industrial package for RDT 2 and RDT 3.

\section{RDT - Armature \& Base}

The RDT 2 armature and base IC chip are shown in Fig. 1 . The sapphire armature disk includes an electrically interconnected metallization pattern divided into a 10-bit Gray code uninterrupted around the entire 360 degrees. Each track of the Gray code has a complementary metallization pattern which provides the differential signal required for the detector pairs, meaning that there is a total of 20 tracks.

The base chip is fabricated using 2.0 micron, 2 metal, single poly-CMOS design rules. It contains two arrays of detectors, a radial and a circumferential array, along with circuits for encoding angular position into a 13-bit binary number and all of the circuitry for 3-wire multiplexing. Each detector reads the signals from a pair of complementary tracks on the armature disk. A ten detector pair radial array reads the 10 complementary tracks on the armature to produce the 10 most significant bits. The outer tracks of the armature and the circumferential detector array are arranged to form a vernier which provides the three least significant bits of the 13-bit value. Unlike UAST 3, which uses convolution to digitize the vernier output, RDT 2 uses a logic array and the output of 16 detector pairs to produce the 3 least significant bits. RDT 3, currently under development, is designed to provide increased resolution (up to 16 bits) and to decrease the required packaging precision.

\section{$\underline{\text { RDT - Performance }}$}

In summary, an RDT capable of achieving 13 bits of resolution (RDT 2) has been demonstrated. A device capable of at least 16 bits resolution is under development. These devices provide absolute encoding of angular position over 360 degrees. The RDT is designed to allow multiplexing of up to 128 sensors on a three-wire bus with a sampling rate of at least 400 samples/sec per sensor.

Continuous rotation life tests have demonstrated operation in excess of $200,000,000$ cycles at $2500 \mathrm{rpm}$. Cycling with $90 \mathrm{deg}$. oscillations and high acceleration at $1 \mathrm{~Hz}$ showed continued operation after 1.2 million cycles. In all 
cases the tests terminated before failure of the device. The maximum speed at which the devices have been operated is approximately $2500 \mathrm{rpm}$, but higher speeds should be possible.

\section{FLUID SHEAR TRANSDUCER (FST)}

\section{FST - Objectives}

Advanced submarine, ship, and aircraft configurations are routinely developed and evaluated using numerical techniques (computational fluid dynamic analyses) which often require experimental validation. Moreover, some of these configurations utilize conformal or adaptive surfaces to actively manage separation, turbulence, boundary layer development, and other features of flow. As such, distributed transducers are needed to detect and measure fluid-induced shear (as well as pressure), in two directions, over the surfaces of scale model and operational vehicles moving in fluid. For example, Fig. 13 illustrates a separated flow over an airfoil where each shear sensor in an array could be used to determine surface flow direction, collectively used to indicate separation, and unsteady flow.

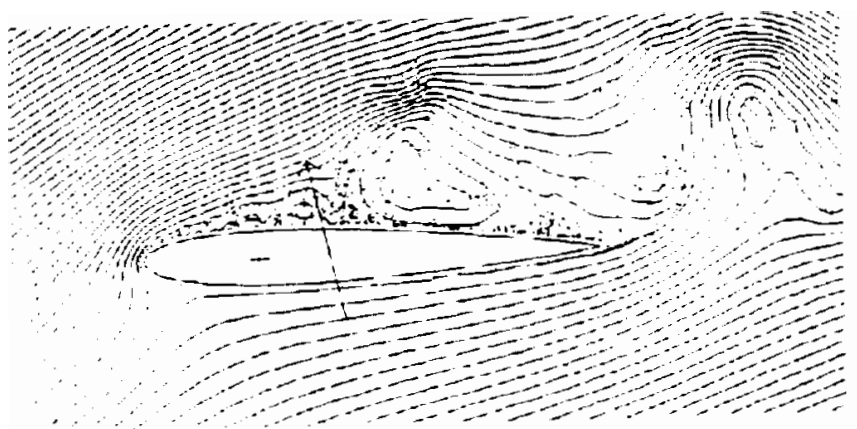

Figure 13. Flow separates on a NACA 0012 airfoil above 16 degrees angle-of-attack for Re numbers above 10,000,000.

\section{FST - Configuration}

Fluid shear transducers (FST), which are based on "direct force" measurements, are a natural extension of the CMOS sensing described in this paper. In order to minimize interaction between the fluid and sensor, as well as sensitivity to body loads such as gravity and vehicle accelerations, the sensing disk (see Fig. 14) must be supported on a stiff suspension which undergoes small translational movements and possesses high rotational stiffness for minimal cross-axis coupling. For example, using a viscous fluid-coupling between the flow and a $1.8 \mathrm{~cm}$ diameter suspended disk, to characterize flows with local Reynolds numbers above $10,000,000$, a sensor must resolve transverse forces on the order of $50 \mu \mathrm{N}(0.2 \mathrm{~Pa}$ of shear) with a dynamic range of $0.8 \mathrm{~N}(3 \mathrm{kPa})$ and be designed to survive substantial overloads. For a suspension with a lateral stiffness of $1,300 \mathrm{~N} / \mathrm{m}$, this corresponds to resolving dimensional deflections of less than 30 Angstroms over a range several times greater than $112 \mu \mathrm{m}$.

Many applications need high spatial and large bandwidth shear measurements which naturally leads to a requirement for multiplexing sensor outputs on to common busses. Also, the sensor networks will have to operate in water at great depths so sealed packages with pressure compensation are often necessary. In addition to solving these and the mechanical suspension and packaging design issues, a CMOS IC sensor is required which can make high resolution bi-axial measurements with a large dynamic range. The resulting bi-axial strain transducer (BiAST) IC chip, presently under development, will work for other applications as well, including measurements of bi-axial strain, acceleration, and others. Development of a three (orthogonal) axis CMOS IC sensing chip (TriAST) design is already underway.

Figure 14 shows the configuration of an individual shear sensor, including details of the viscous shear-coupled disk, sensor housing, suspension rods which allow controlled translation of the disk and armature with low vertical deflection, welded bellows to permit relative translational motion between the disk and housing (with high rotational stiffness to prevent cross-axis coupling) while maintaining a hermetic seal between the sensor interior and the fluid environment, armature and base chip at the bottom of the housing which measure armature translation in two dimensions, and sensor receptacle (sensor/receptacle size is $19 \mathrm{~mm} \mathrm{dia}$. and $18 \mathrm{~mm}$ in height). The suspension facilitates a controlled spacing between the base and armature of approximately $12 \mu \mathrm{m}$. The system is temperaturecompensated by the suspension configuration and compatibility of materials.

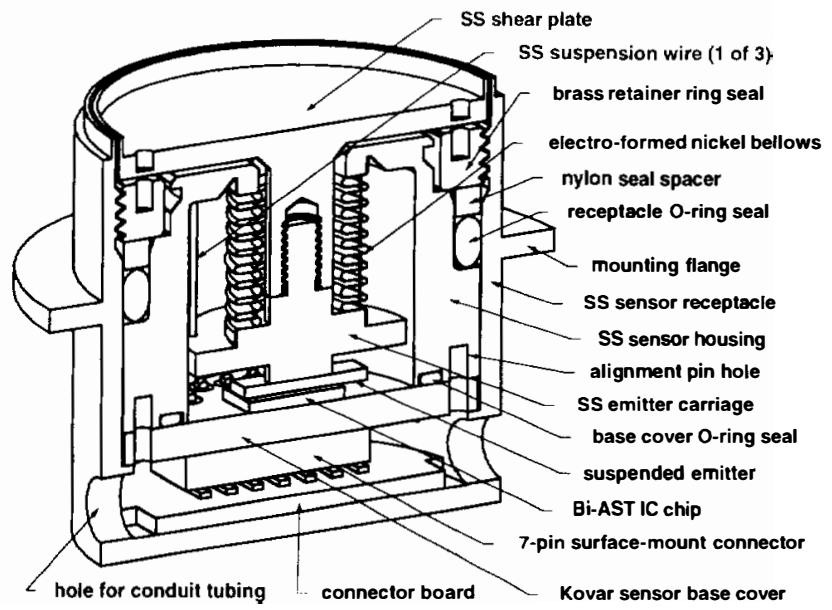

Figure 14. Cutaway illustration of the FST sensor module and receptacle.

Figure 15 shows a string of "vaulted" (sealed) sensors where mixed types of transducers (some are two-axis shear, others are pressure, and a thin film anemometer module is also shown). All sensors share a common sensor receptacle and network conduit interface. In addition to each sensor module being hermetically sealed, the conduit tubing and receptacles form a separate control volume which can be gas-filled and pressure compensated (via a bellows with sufficient starting volume to accommodate the anticipated increase in external water pressure) to prevent intrusion of water when operating the network at great pressures. The sensor modules utilize standardized connectors and O-ring seals to facilitate easy removal or to reconfigure the network with different combinations of sensor types for various applications.

\section{FST - Armature \& Base}

At the heart of the two-axis surface shear stress sensor is the BiAST, which uses the CMOS IC chip layout shown in Fig. 16. Again, like the UAST, the BiAST is really a microextensometer, not a strain transducer (the sensor base or 
armature are not distorted but, rather, sensor output is based on measured displacements between the base and armature). The BiAST base chip includes two orthogonal sets of 64-element detector arrays interacting with a suitably designed, driven set of orthogonal emitter arrays. Together these form two separate verniers which can be used to make measurements for the two inplane axes. The detector and emitter element spacing are the same as those for the UAST 3 (55.8 and $57.6 \mu \mathrm{m}$, respectively) and the BiAST shares the eight analog-to-digital converters, template generator, and strain computation engine of the UAST 3 between the two arrays. As such, the BiAST first samples both detector arrays and then begins conversion of the $\mathrm{X}$-axis detectors with subsequent calculation of the $\mathrm{X}$-axis displacement. The $\mathrm{Y}$-axis detector values are then converted with subsequent calculation of the $\mathrm{Y}$-axis value. Thus, the BiAST can sample only half as fast as the UAST for the same level of output resolution. Otherwise, its performance is expected to be on par with that of the UAST 3.

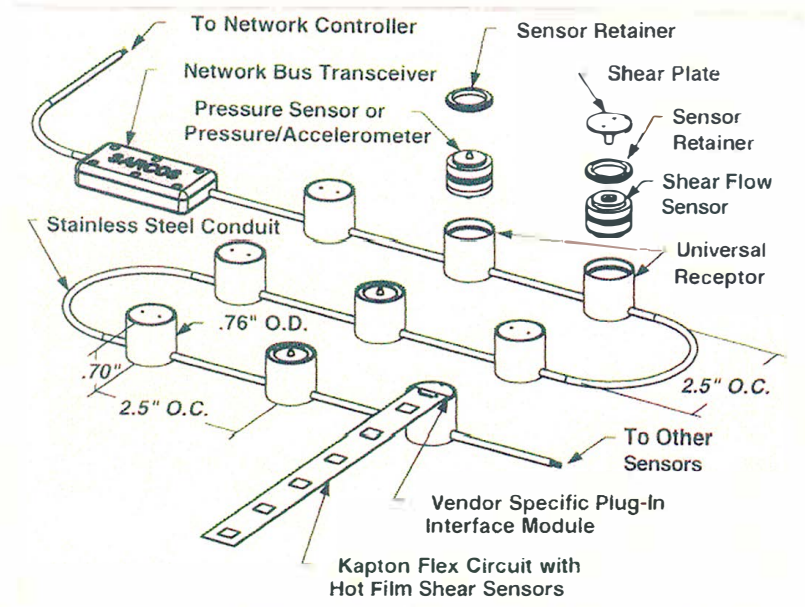

Figure 15. Shown are a number of interconnected standard packages configured with different sensors. Included are: twoaxis flush-mounted flow sensors, pressure sensors, and a hot film anemometer strip.

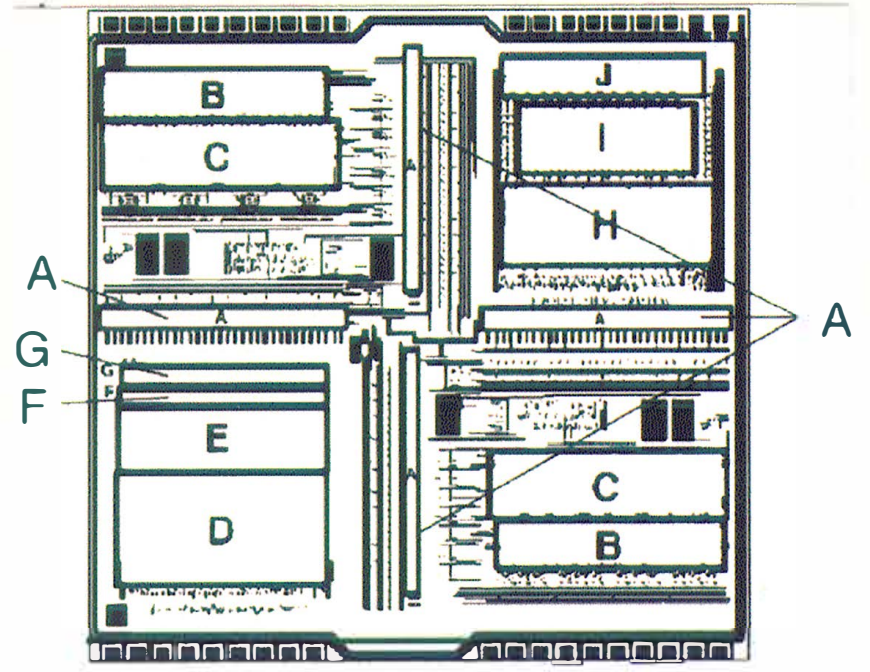

Figure 16. Preliminary IC chip layout of the BiAST presently under development. A) $32 \mathrm{ea}$. integrating detectors; B) 4 ea. Delta-Sigma modulators; C) 4 ea. accumulators; D) multiplieraccumulator; E) template generator; $F)$ binary search registers; G) binary search control; H) EEPROM + new functions; I) $64 x$ 12 RAM; and J) token interface + BiAST control.
PRESSURE TRANSDUCER NETWORK (PTN)

\section{PTN - Obiectives}

In addition to shear stress measurements, pressure distribution data with high spatial density and large sample rates are essential to characterize flow features around hydrodynamic surfaces, such as submarine control surfaces, propulsor and hull; aircraft foils and adaptive surfaces; turbine blades; and others. With this in mind, integrated networks of micro pressure transducers tailored for applications in demanding environments, such as aircraft, submarines, and other systems, have been developed.

For example, Fig. 17 shows a network of pressure and vibration sensors integrated on both faces of a prototype 1/4scale propulsor blade section. This network consists of 72 sensors housed in 56 hermetic packages designed for use in different environments. More specifically, the network is divided into two major branches, installed on opposite faces of the blade and comprised of: (1) 8 nodes which perform analogto-digital conversion and transmit data over a digital bus at a combined rate of $12 \mathrm{Mbits} / \mathrm{sec}$; (2) 8 high-speed pressure sensors (sampled at $50 \mathrm{kHz}$ ); (3) 32 low speed-sensors (sampled at $5 \mathrm{kHz}$ ); and (4) 32 collocated pressure and vibration sensors (also sampled at $5 \mathrm{kHz}$ ). The CMOS base/armature concept outlined throughout this paper was readily combined with bulk silicon micro-machining techniques and better-than-hermetic bonding methods to fabricate the sensors used in this example.

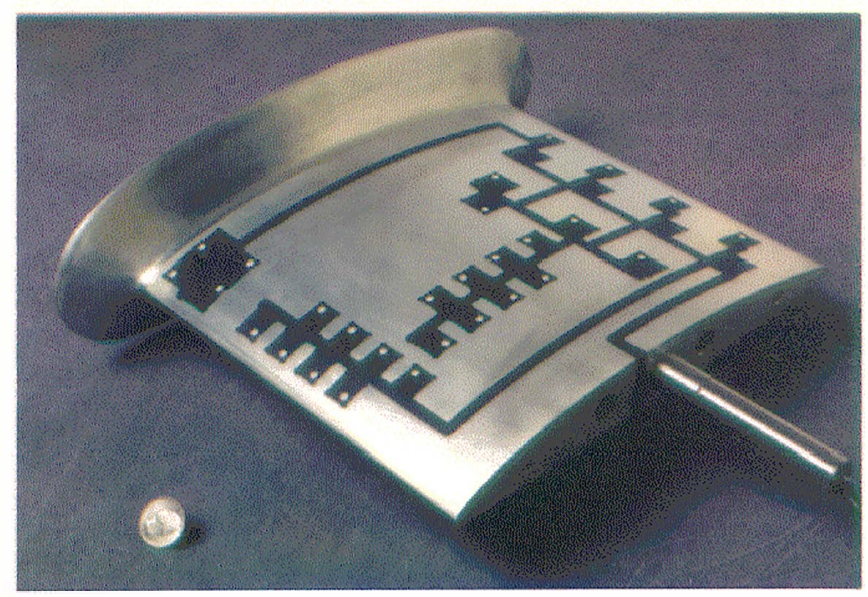

Figure 17. Propulsor test blade showing the layout of the pressure and vibration sensor network.

\section{PTN - Configuration}

Figure 18 illustrates a hermetically sealed, tlat pressure sensor package in which the sensing diaphragm is placed in direct contact with the environment and where the electrical conduits are sealed in metallic tubes in a way that is similar to that used for the shear stress sensor network described in the previous section.

In the PIN sensor package, a silicon sensing diaphragm covered with a low stress silicon nitride film is eutectically bonded to a 1 -to- $2 \mathrm{~mm}$ thick Pyrex ${ }^{\circledR}$ plate, and the latter is then eutectically bonded to a plated Kovar case. Kovar tubes are brazed on the case and act as vaults for the network electrical conduits. Electrical connection between the IC chip and the network bus is provided by a flexible Kapton ${ }^{\circledR}$ conduit which is flip-chip bonded to the IC chip. Passive electronic components, 
such as filter capacitors and bias resistors are mounted on the Kapton ${ }^{\circledR}$ flexible conduit and the latter, is then, attached to the lid. Electrical insulation and passivation of the diaphragm and housing are provided by low stress silicon nitride (deposited on the diaphragm) and by a 15 to $25 \mu \mathrm{m}$ thick Parylene conformal coating over the entire package.

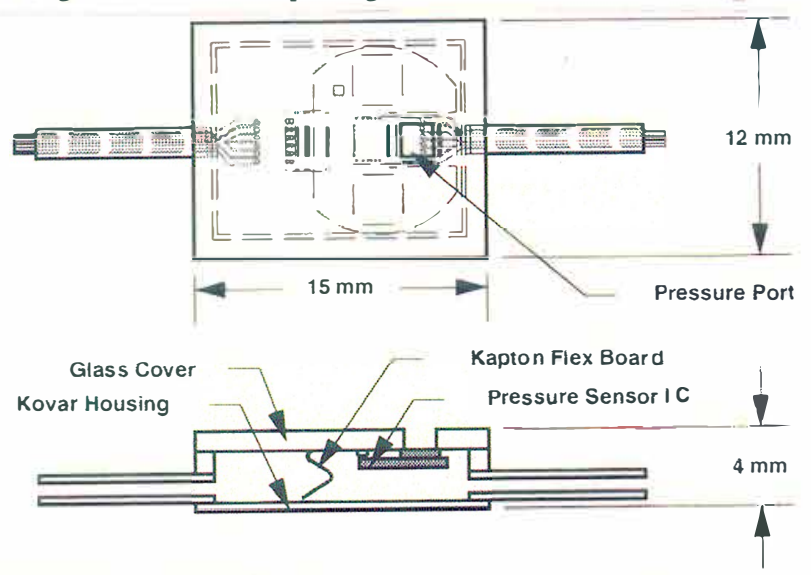

Figure 18. Schematic representation of a new type of pressure and vibration sensors package.

\section{PTN - Armature \& Base}

Similar to the other sensors described throughout this paper, the pressure sensors exploit the field-based (base/armature) sensing technique [1-5] and a concept called the "block approach", where bulk micro-machined elements such as pressure-sensing diaphragms or proof masses are joined (in multiple layers if necessary) to conventionally fabricated VLSI CMOS IC chips to form complete transducers.

Figure 19 shows a micrograph of a typical pressure sensing CMOS IC chip (base) and pressure-sensing diaphragm (armature). Each base IC chip ( $2.2 \times 4.4 \times 0.5 \mathrm{~mm})$ features: (1) a differential pair of field-based sensors, with one detector pad located toward the center of the diaphragm (maximum deflection area) and one located at a corner (reference sensor) to minimize temperature effects and variation in the assembly gap, (2) a contact and bonding aluminum frame to which the sensing diaphragm is attached, (3) a 5-pole Chebychev switchedcapacitance low-pass filter, and (4) multiplexing and bus driver electronics. In principle, an array of detectors, located on the base, could be used to enhance resolution and, to respond to the mechanical dynamics of the membrane.

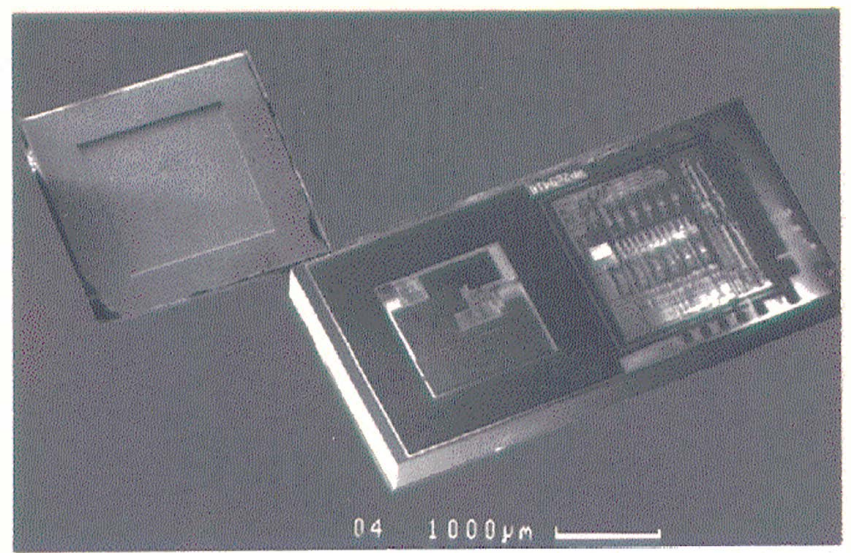

Figure 19. Micrograph showing a typical pressure sensor CMOS IC base chip and a diaphragm.
The pressure sensing diaphragm used as the armature in these devices consists of: (1) a contact and bonding outer frame (approx. $2350 \times 2350 \mu \mathrm{m}$ wide), and (2) a square diaphragm area (approx. $1500 \times 1500 \mu \mathrm{m}$ wide) of variable thickness. The diaphragms are RIE micro-machined out of $50 \mu \mathrm{m}$ thick, n-type single crystal silicon membranes, metallized and then hermetically sealed, under vacuum, to the CMOS base chip.

\section{PTN - Performance}

Pressure sensors developed to date have been tailored to operate over the range from 0 to approximately $3 \mathrm{~atm}$. and have a spectral noise density of approximately $1.5 \mathrm{~Pa} / \mathrm{Hz}^{1 / 2}$ (i.e., approx. 10 bits of dynamic range for the slow pressure sensor). Shock tube tests have demonstrated the capability of the devices to accurately monitor transient pressure variations with characteristic frequencies above $20,000 \mathrm{~Hz}$. In practice, the diaphragm may be designed to adjust the dynamic range as dictated by the application. It is also anticipated that enhanced pressure resolution could be achieved by replacing the synchronously demodulated field-based sensor, used in the current device, by the charge-integrating amplifier developed for the UAST and the BiAST.

\section{ACKNOWLEDGMENTS}

The authors gratefully acknowledge the direct contributions from D.P. Marceau, D.L. Wells, F.L. Williams C.C. Davis, D.G. Petelenz, and D. Beutel. The authors also gratefully acknowledge support for this effort by Defense Advanced Research Projects Agency under contract numbers: F33615-87-C-5267, N00014-93-C-0112, N00014-96-C-0345, DAAH01-95-C-R144， DABT63-95-C-0033， N00014-93-C0181, from NAVSEA under Newport News Shipbuilding (N00024-95-C-2102) as well as from DARPA and NASA funded projects through Martin Marietta, McDonnell Douglas and Boeing

\section{REFERENCES}

1. B.J. Maclean, M.G. Mladejovsky, M.R. Whitaker, M. Olivier and S.C. Jacobsen, "A Digital MEMS-Based Strain Gage for Structural Health Monitoring", in Proceedings of Material Research Society Symposium, Boston, MA, Dec. 1-5 (1997).

2. S.C. Jacobsen, et. al., "Field-based Microsystems for Strain Measurement", Proc. ASME Winter Annual Meeting, November, Chicago, IL (1988).

3. S.C. Jacobsen, et. al. "Displacement Sensing by Direct Mechanical Modulation of Shaped Electro-Active MicroStructures", Proc. IEEE Micro-Electro Mechanical Systems Workshop, Feb. 11-14, Napa Valley, CA (1990).

4. S.C. Jacobsen, et. al., "Field-Based State Sensing in MicroMotion Systems", in Integrated Micro-Motion Systems. Micromachining, Control, and Applications, F. Harashima, Ed., Elsevier Science Publishers (1990).

5. S.C. Jacobsen, et. al., "Advanced Intelligent Mechanical Sensors (AIMS)", Proc. IEEE Transducers '91, June 24-28, San Francisco, CA (1991). 